One threat, different answers: the impact of COVID-19 pandemic on cornea donation and donor selection across Europe (see page 312)

In this survey we found that the decrease in transplant activity during the pandemic peak varied between countries in parallel with the stringency of official recommendations for donor selection, regardless of the severity of the pandemic.

Effect of herpes simplex keratitis scar location on bilateral corneal nerve alterations: an in-vivo confocal microscopy study (see page 319)

The location of corneal HSV scars induces differential corneal nerve and sensation loss, with central scars causing more nerve damage than peripheral scars. Scar location may be an indicator for risk of neurotrophic keratopathy.

Outcomes of corneal neurotisation using processed nerve allografts: a multicentre case series (see page 326)

Patients with neurotrophic keratopathy achieve good recovery of corneal sensation after neurotisation with processed acellular nerve allografts.

Epidemic keratoconjunctivitis in India: electronic medical records driven big data analytics report IV (see page 331)

A retrospective study of 19203 patients with acute epidemic keratoconjunctivitis found that it was a self-limiting disorder which more commonly affected males and was unilateral. Corneal involvement occurred in about a third of patients.

Stromal peeling for deep anterior lamellar keratoplasty in post-penetrating keratoplasty eyes (see page 336)

In eyes that have undergone penetrating keratoplasty (PK) for keratoconus, the corneal stroma can be successfully exchanged through large diameter keratoplasty using simple stromal peeling, which does not require deep anterior lamellar dissection of the in situ PK graft.

Influence of age on small incision lenticule extraction outcomes (see page 341)

SMILE is effective, safe and relatively predictable for myopia management in patients over 40 years of age. However, refractive outcomes are worse than in younger patients, with a tendency to astigmatic undercorrection.

Anterior chamber depth, lens thickness and intraocular lens calculation formula accuracy: a comparison of nine formulae (see page 349)

Anterior chamber depth and lens thickness may significantly influence postoperative refractive results, independently of the axial length. New generation formulae are less influenced by both, and a better choice in particular for eyes with extreme biometric values.

Health-related quality of life in Ahmed FP7, and Baerveldt 250 and 350 patients compared with medical controls (see page 356)

Health-related quality of life was similar among Ahmed FP7, Baerveldt 250 and Baerveldt 350 device groups, but Baerveldt 350 patients had lower adult Strabismus-20 questionnaire self-perception scores than controls managed medically, suggesting greater implant visibility or awareness.

Outcomes and risk factors for failure after trabeculectomy in Taiwanese patients: medical chart reviews from 2006 to 2017 (see page 362)

Of the 237 initial trabeculectomies, $67.1 \%$ of the patient maintained qualified intraocular pressure control at 10 year follow-up in a Taiwanese population. Poor preoperative visual acuity and young age were risk factors for surgical failure

Clinical profile and outcome of early surgery in neonatal-onset glaucoma presenting over a 5 -year period (see page 368)

Fifty-three neonatal-onset glaucoma infants (94 eyes) who underwent early surgery were studied. $66 \%$ had primary congenital glaucoma; $45.7 \%$ required additional surgery. $28.3 \%$ had good vision (LogMAR $>0.4$ ) and $34.7 \%$ had moderate impairment (LogMAR $0.5-1.0)$.

Cascade screening for glaucoma in high-risk family members of AfricanCaribbean glaucoma patients in an urban population in London (see page 376) First-degree relatives of African-Caribbean patients are at high risk of developing glaucoma. A cascade screening study was performed to identify undiagnosed glaucoma in this population. This strategy failed, with poor participation and no new glaucoma cases.

Normative profiles of neuroretinal rim area in a multiethnic Asian population: the Singapore epidemiology of eye diseases study (see page 381)

Fifth percentile values for HD-OCT neuroretinal rim area were significantly different between ethnicities in this multi-ethnic Asian study, emphasising the need for ethnicity-specific normative databases.

Convolutional neural network to identify symptomatic Alzheimer's disease using multimodal retinal imaging (see page 388)

A convolutional neural network using multimodal retinal images (optical coherence tomography (OCT), OCT angiography, and ultra-widefield scanning laser ophthalmoscopy) and patient data inputs was created to distinguish individuals with Alzheimer's disease from cognitively healthy adults.

Deep learning-based classification and segmentation of retinal cavitations on optical coherence tomography images of macular telangiectasia type 2 (see page 396)

A fully-automatic deep learning-based method was developed to classify and segment retinal cavitations on OCT images of MacTel2. The method was computationally efficient and demonstrated good performance.

Randomised study evaluating the pharmacodynamics of emixustat hydrochloride in subjects with macular atrophy secondary to Stargardt disease (see page 403)

In this randomised, double-masked study, emixustat hydrochloride demonstrated dose-dependent suppression of rod b-wave amplitude recovery postphotobleaching, confirming emixustat's biological activity in patients with macular atrophy secondary to Stargardt disease 


\section{At a glance}

Irish national diabetic RetinaScreen programme: report on five rounds of retinopathy screening and screen-positive referrals (INDEAR study report No. 1) (see page 409)

Uptake of screening has improved in Ireland. More than 100000 individuals were screened annually in the rounds four and five. The referrable retinopathy detection rate has reduced since the introduction of screening in Ireland.

Microvascular abnormalities and long-term efficacy after stereotactic radiotherapy under continued intravitreal anti-VEGF treatment for neovascular AMD (see page 415)

Besides an association with reduced need for ranibizumab/aflibercept treatment through years 2 and 3 after low-energy stereotactic radiotherapy in neovascular age-related macular degeneration, we observed a visual acuity reduction and high numbers of microvascular abnormalities.

Characterisation of macular superficial vessel density alteration in preclinical ethambutol-induced optic neuropathy using optical coherence tomography angiography (see page 422)

Macular vessel density decreased and mGCIPL and pRNFL thicknesses remained unchanged in patients using ethambutol for 6 months. cCVD might be an earlier indicator for monitoring early-stage ethambutol toxicity than mGCIPL thickness.

Visual impairment and perceptual visual disorders in children with cerebral palsy in Nigeria (see page 427)

$51 \%$ of children with cerebral palsy had ocular abnormalities, almost half had cerebral visual impairment. Visual dysfunction was associated with oculomotor dysfunction. Only $14 \%$ of carers recognised visual problems, highlighting the need for greater awareness.

Time trends, associations and global burden of intraocular foreign bodies (see page 435 )

The global total of disability-adjusted life years due to IOFBs rose by $43.7 \%$ between 1990 and 2017. Country-level disease burden due to IOFBs was closely associated with lower socioeconomic level and prevalence of glaucoma.

In-vitro establishment, validation, and characterisation of conjunctival epithelium outgrowth using tissue fragments and amniotic membrane (see page 440)

In vitro validation of outgrowth of conjunctival cells from conjunctival tissue fragments glued over the amniotic membrane for expansion of the conjunctival epithelium. 